\title{
Herpes Simplex Virus Encephalitis: Patterns of Epidemiology and Outcomes of Patients Admitted to the Intensive Care Unit in Texas, 2008 - 2016
}

\author{
Lavi Oud
}

\begin{abstract}
Background: Patients with herpes simplex virus encephalitis (HSVE) often require admission to the intensive care unit (ICU) and have considerably worse outcomes than those not critically ill. The short-term outcomes of critically ill patients in the general population have markedly improved over the past decades. However, the population-level patterns of demand for critical care services among patients with HSVE have not been examined, and it is unknown whether there were corresponding outcome gains among those admitted to the ICU.
\end{abstract}

Methods: The Texas Inpatient Public Use Data File was used to identify hospitalizations with HSVE aged $\geq 18$ years during $2008-2016$. ICU admissions were identified using unit-specific revenue codes. The patterns of ICU utilization and those of short-term outcomes (with short-term mortality defined as in-hospital death or discharge to hospice) were examined across demographic strata and over time.

Results: Among 1,964 hospitalizations with HSVE, 1,176 (59.9\%) were admitted to ICU $(45.8 \%$ aged $\geq 65$ years; $53.1 \%$ female, among ICU admissions). ICU utilization increased with age (from $47.9 \%$ (age 18 - 44 years) through $61.2 \%$ (older adults (age $\geq 65$ years)); $\mathrm{P}$ $=0.0003$ for trend), and increased over time only among older adults (odds ratio: 1.06/year (95\% confidence interval (CI): 1.01 - 1.12)). Among ICU admissions, routine home discharge, transfer to a postacute care facility, and short-term mortality occurred in $26.8 \%, 39.5 \%$, and $18.7 \%$, respectively; the corresponding outcomes for older adults were $10.6 \%, 51.4 \%$, and $26.2 \%$, respectively. The outcomes for the whole cohort of ICU admissions remained unchanged over time.

Conclusions: Adults with HSVE had high demand for critical care services, and those admitted to ICU had high short-term mortality and substantial residual morbidity among survivors, which remained unchanged over time. These findings can inform clinicians' decisionmaking and discussions about goals of care with affected patients and their surrogates.

Manuscript submitted October 14, 2019, accepted October 31, 2019

Division of Pulmonary and Critical Care Medicine, Department of Internal Medicine, Texas Tech University Health Sciences Center at the Permian Basin, 701 W. 5th St., Odessa, TX 79763, USA.Email: lavi.oud@ttuhsc.edu

doi: https://doi.org/10.14740/jocmr4025
Keywords: Herpes simplex virus; Encephalitis; Intensive care unit; Epidemiology; Short-term outcomes

\section{Introduction}

Herpes simplex virus (HSV) is the most common cause of sporadic encephalitis [1], and it accounts for $30-40 \%$ of encephalitis cases where an etiologic diagnosis is made [2]. The introduction of antiviral therapy with acyclovir in the mid-1980s has reduced early mortality from about $70 \%$ [3] to generally less than $10 \%$ [4]; and the subsequent advent of deoxyribonucleic acid (DNA) amplification by polymerase chain reaction, introduced in the early 1990s [5] has markedly enhanced the recognition of affected patients. Nevertheless, residual morbidity with moderate to severe neurological deficits continues to affect survivors of herpes simplex virus encephalitis (HSVE), ranging from $43 \%$ [6] to $58 \%$ [7].

Patients with HSVE often develop complications requiring care in the intensive care unit (ICU). These critically ill patients are expected to have a higher risk of short-term mortality and to have more commonly severe residual morbidity sequelae. Numerous studies have described the epidemiology and outcomes of hospitalized patients with HSVE [4-6]. However, there have been only few studies that specifically examined critically ill patients with HSVE that require ICU admission. In two recent multicenter studies from France, ICU admission was reported in $32.8 \%$ [8], and mortality among ICU admissions ranged from $11.9 \%$ at the end of hospitalization [8] to $17 \%$ at 90 days [9]. However, the patterns of demand for critical care services among patients with HSVE and longitudinal trends of hospital outcomes among the critically ill have not been reported, to our knowledge. In addition, although improving care of the critically ill has been associated with progressively reduced hospital mortality of ICU patients in the general population [10], it is unknown whether these outcome gains extended to critically ill patients with HSVE over time.

Contemporary data on the epidemiology and outcomes of patients with HSVE requiring admission to the ICU can inform clinicians on their evolving need for critical care resources, better characterize the impact of the current interventional efforts, and provide benchmarking for future performance improvement efforts. 
Here, we report a population-based cohort study of hospitalized adults with HSVE to characterize the spatial and temporal patterns of: 1) The demand for critical care resources; and 2) Hospital outcomes of those hospitalizations admitted to the ICU, for the whole cohort and across demographic strata.

\section{Materials and Methods}

This was a retrospective, population-based cohort study. Because we used a publicly available, de-identified data set, the study was determined to be exempt from formal review by the Texas Tech Health Sciences Center's Institutional Review Board.

\section{Data sources and study population}

We used the Texas Inpatient Public Use Data File (TIPUDF), an administrative data set maintained by the Texas Department of State Health Services [11]. The use of TIPUDF has been previously described [12]. Hospitalizations with HSVE aged 18 years or older during the years 2008 - 2016 were identified using International Classification of Diseases, Ninth Revision (ICD-9), Clinical Modification code 054.3 and International Classification of Diseases, Tenth Revision (ICD-10), Clinical Modification B00.4. All the identified HSVE hospitalizations were included in the study analyses. Because TIPUDF provides discharge-level, rather than patient-level information, precluding accounting for repeated admissions in the data set, we report the number of hospitalizations and ICU admissions as units of analysis, rather than number of patients. Hospitalizations with ICU admission were identified based on unit-specific revenue codes for an ICU.

\section{Outcomes}

The primary outcome was the rate of short-term mortality among hospitalizations with HSVE admitted to the ICU. Short-term mortality was defined as that of hospitalizations with in-hospital death or discharge to hospice.

The secondary outcomes included: 1) Rates of admission to the ICU among HSVE hospitalizations; 2) Hospital disposition of ICU admissions without in-hospital death or discharge to hospice. Among these survivors we focused further on those with routine home discharge and those discharged to post-acute care facilities. The latter discharge destinations were selected as claim-based data proxies for the best short-term outcome and presence of substantial residual morbidity, severe enough to require ongoing inpatient care, respectively.

\section{Study variables}

The extracted covariates included age, gender, hospital disposition and year of admission. Hospital disposition was categorized as home (with subcategories of routine home and home with health care services), post-acute care facility (which included long-term care hospital, skilled nursing facility, and inpatient rehabilitation), another acute care facility, in-hospital death, and hospice.

\section{Data analysis}

The characteristics of HSVE hospitalizations are presented as numbers and percentages for categorical variables.

Trends of ICU admission rates and of specific categories of hospital disposition across age groups were examined using the Cochran-Armitage test. Chi-square test was used to assess gender-associated differences in ICU admission rates and hospital disposition.

Temporal trends of ICU utilization and individual hospital disposition categories for the whole cohort and within specific age and gender strata were examined using univariate logistic regression, with the year of admission used as independent variable. Regression findings of the annual change for each outcome were expressed as odds ratio (OR) per year and $95 \%$ confidence intervals $(95 \% \mathrm{CI})$.

Data management was performed using Excel and Access (Microsoft, Redmond, Washington) and statistical analyses were performed with MedCalc version 17.5.5 (MedCalc Software, Ostend, Belgium). A two-sided P value $<0.05$ was considered statistically significant.

\section{Results}

There were 1,964 hospitalizations with HSVE in 335 hospitals during the study period, of which 1,176 (59.9\%) were admitted to ICU. The rate of ICU admission rose with age, from $47.9 \%$ among those aged 18 - 44 years to $61.2 \%$ among those 65 years or older $(\mathrm{P}=0.0003$ for trend). The rate of admission to ICU was higher among males, but the difference was not statistically significant $(61.1 \%$ vs. $56.9 \%$, respectively; $\mathrm{P}$ $=0.0786)$. The rate of ICU admission did not change over the study period for the whole cohort of HSVE hospitalizations (OR: 1.04 (95\% CI: 0.99 - 1.07); $\mathrm{P}=0.0548)$, but rose progressively among males (OR: 1.08 (95\% CI: $1.02-1.14) ; \mathrm{P}=$ $0.0104)$ and those 65 years or older (OR: 1.06 (95\% CI: 1.01 - 1.12); $\mathrm{P}=0.0383$ ). The rate of ICU admission did not change significantly over time among the other age groups or among females (data not shown).

The characteristics of the cohort are detailed in Table 1. Among HSVE hospitalizations admitted to ICU 539 (45.8\%) were 65 years or older, and 537 (53.1\%) were female. ICU admissions were older than those not requiring ICU care and had substantially worse outcomes across all categories of hospital disposition, except transfers to another acute care hospital. Thus, home discharge was markedly lower among ICU admissions, as compared to those without ICU admission $(36.5 \%$ vs. $60.9 \%$, respectively), while discharge to a post-acute care facility was markedly higher (39.5\% vs. $25.5 \%$, respectively). The short-term mortality was markedly higher among ICU admissions ( $18.7 \%$ vs. $4.6 \%$, respectively). 
Table 1. The Characteristics of Hospitalizations With Herpes Simplex Virus Encephalitis

\begin{tabular}{|c|c|c|c|}
\hline \multirow{2}{*}{ Category } & All & $\mathrm{ICU}^{\mathrm{a}}$ & Non-ICUb \\
\hline & $N=1,964$ & $N=1,176$ & $N=788$ \\
\hline \multicolumn{4}{|l|}{ Age (years) } \\
\hline $45-64$ & $674(34.3)$ & $441(37.5)$ & $233(29.6)$ \\
\hline$\geq 65$ & $881(44.9)$ & $539(45.8)$ & $342(43.4)$ \\
\hline Male & $776(45.1)$ & $474(46.9)$ & $302(42.6)$ \\
\hline \multicolumn{4}{|l|}{ Hospital disposition } \\
\hline All home & 909 (46.3) & $429(36.5)$ & $480(60.9)$ \\
\hline Home routine & $648(33)$ & $315(26.8)$ & $333(42.3)$ \\
\hline Death $^{\mathrm{f}}$ or hospice & $256(13)$ & $220(18.7)$ & $36(4.6)$ \\
\hline Death & $172(8.8)$ & $146(12.4)$ & $26(3.3)$ \\
\hline Hospice & $84(4.2)$ & $74(6.3)$ & $10(1.3)$ \\
\hline
\end{tabular}

aCU: hospitalizations with admission to ICU. bNon-ICU: hospitalizations without admission to ICU. 'Gender was reported for 1,720 of all hospitalizations, 1,011 of ICU admissions and 709 of those without ICU; the percent figures for gender in each column refer to that column's denominator for gender. ${ }^{d}$ Home health denotes those hospital discharges requiring home health services. ${ }^{e}$ Post-acute care denotes long-term care hospitals, skilled nursing facilities, and inpatient rehabilitation. fDeath denotes hospitalizations with in-hospital death. ICU: intensive care unit.

The patterns of specific hospital disposition categories within the age and gender strata of HSVE hospitalizations admitted to ICU are detailed in Table 2. The rates of routine home discharge have decreased precipitously with age (50.5\% among those aged 18 - 44 years vs. $10.6 \%$ among those 65 years or older; $\mathrm{P}<0.0001$ for trend), while the rates of discharge to a post-acute care facility, in-hospital death, discharge to hospice and overall short-term mortality all rose with age. On the other hand, hospital disposition destinations did not dif- fer across gender categories. HSVE hospitalizations involved admission to ICU in $84.9 \%$ of all hospital deaths and $85.9 \%$ of all short-term mortality events.

The temporal trends of hospital disposition categories among ICU admissions are detailed in Table 3. Generally, there was no significant change over time in home discharge, transfers to post-acute care facilities, transfers to another acute care hospital or short-term mortality, except in selected groups. The rate of all home discharges among females decreased be-

Table 2. Hospital Disposition Among Hospitalizations With Herpes Simplex Virus Encephalitis Admitted to ICU, Stratified by Age and Gender

\begin{tabular}{|c|c|c|c|c|c|c|c|}
\hline \multirow{3}{*}{ Category } & \multicolumn{3}{|c|}{ Age } & \multicolumn{4}{|c|}{ Gender } \\
\hline & \multirow{2}{*}{$\begin{array}{l}18-44 \\
N=196\end{array}$} & \multirow{2}{*}{$\begin{array}{l}45-64 \\
N=441\end{array}$} & \multirow{2}{*}{$\begin{array}{l}\geq 65 \\
N=539\end{array}$} & \multirow[t]{2}{*}{ P value } & \multirow{2}{*}{$\begin{array}{l}\text { Female } \\
\mathbf{N}=\mathbf{5 3 7}\end{array}$} & \multirow{2}{*}{$\begin{array}{l}\text { Male } \\
N=474\end{array}$} & \multirow[t]{2}{*}{ P value } \\
\hline & & & & & & & \\
\hline All home & $118(60.2)$ & 209 (47.4) & $102(18.9)$ & $<0.0001$ & $179(33.3)$ & $169(35.7)$ & 0.4385 \\
\hline Home routine & $99(50.5)$ & $159(36.1)$ & $57(10.6)$ & $<0.0001$ & $131(24.4)$ & $115(24.3)$ & 0.9607 \\
\hline Home health ${ }^{\mathrm{a}}$ & $19(9.7)$ & $50(11.3)$ & $45(8.3)$ & 0.3308 & $48(8.9)$ & $54(11.4)$ & 0.1963 \\
\hline Post-acute care ${ }^{\mathrm{b}}$ & $38(19.4)$ & $149(33.8)$ & $277(51.4)$ & $<0.0001$ & $224(41.7)$ & $191(40.3)$ & 0.6476 \\
\hline Another short-term hospital & $15(7.70$ & $19(4.3)$ & $18(3.3)$ & 0.0188 & $22(4.1)$ & $20(4.2)$ & 0.9224 \\
\hline Death $^{\mathrm{c}}$ or hospice & $19(9.7)$ & 60 (13.6) & $141(26.2)$ & $<0.0001$ & $108(20.1)$ & $89(18.8)$ & 0.5929 \\
\hline Death $^{\mathrm{c}}$ & $17(8.7)$ & $37(8.4)$ & $92(17.1)$ & 0.0001 & $70(13)$ & $61(12.9)$ & 0.9374 \\
\hline Hospice & $2(1)$ & $23(5.2)$ & $49(9.1)$ & $<0.0001$ & $38(7.1)$ & $28(5.9)$ & 0.4529 \\
\hline
\end{tabular}

aHome health denotes those hospital discharges requiring home health services. bPost-acute care denotes long-term care hospitals, skilled nursing facilities, and inpatient rehabilitation. 'Death denotes hospitalizations with in-hospital death. ICU: intensive care unit. 
Table 3. Logistic Regression Analyses of the Temporal Trends of Hospital Disposition of ICU Admissions With Herpes Simplex Encephalitis for the Whole Cohort and Across Demographic Strata

\begin{tabular}{|c|c|c|}
\hline Hospital disposition & Odds ratio a $(95 \%$ CI $)$ & $\mathbf{P}$ \\
\hline \multicolumn{3}{|l|}{ All home } \\
\hline All & $0.958(0.914-1.005)$ & 0.0805 \\
\hline \multicolumn{3}{|l|}{ Age (years) } \\
\hline $18-44$ & $0.987(0.880-1.106)$ & 0.8203 \\
\hline $45-64$ & $0.970(0.899-1.046)$ & 0.4351 \\
\hline$\geq 65$ & $0.939(0.862-1.023)$ & 0.1545 \\
\hline \multicolumn{3}{|l|}{ Gender } \\
\hline Female & $0.925(0.860-0.994)$ & 0.0356 \\
\hline Male & $1.006(0.933-1.085)$ & 0.8676 \\
\hline \multicolumn{3}{|l|}{ Home routine } \\
\hline All & $0.937(0.890-0.987)$ & 0.0142 \\
\hline \multicolumn{3}{|l|}{ Age (years) } \\
\hline $18-44$ & $0.898(0.802-1.006)$ & 0.0635 \\
\hline $45-64$ & $0.970(0.897-1.050)$ & 0.4593 \\
\hline$\geq 65$ & $0.917(0.823-1.023)$ & 0.1219 \\
\hline \multicolumn{3}{|l|}{ Gender } \\
\hline Female & $0.906(0.836-0.981)$ & 0.0154 \\
\hline Male & $0.986(0.906-1.073)$ & 0.7434 \\
\hline \multicolumn{3}{|l|}{ Home health $^{\mathrm{b}}$} \\
\hline All & $1.033(0.956-1.117)$ & 0.4053 \\
\hline \multicolumn{3}{|l|}{ Age (years) } \\
\hline $18-44$ & $1.355(1.081-1.697)$ & 0.0083 \\
\hline $45-64$ & $0.993(0.882-1.118)$ & 0.9136 \\
\hline$\geq 65$ & $0.982(0.869-1.109)$ & 0.7694 \\
\hline \multicolumn{3}{|l|}{ Gender } \\
\hline Female & $1.011(0.897-1.139)$ & 0.8560 \\
\hline \multicolumn{3}{|l|}{ Male } \\
\hline \multicolumn{3}{|l|}{ Post-acute care ${ }^{\mathrm{c}}$} \\
\hline All & $1.018(0.971-1.067)$ & 0.4485 \\
\hline \multicolumn{3}{|l|}{ Age (years) } \\
\hline $18-44$ & $0.963(0.836-1.108)$ & 0.6007 \\
\hline $45-64$ & $1.011(0.933-1.095)$ & 0.7804 \\
\hline$\geq 65$ & $1.026(0.959-1.098)$ & 0.4501 \\
\hline \multicolumn{3}{|l|}{ Gender } \\
\hline Female & $1.038(0968-1.112)$ & 0.2876 \\
\hline Male & $0.992(0.922-1.069)$ & 0.8482 \\
\hline \multicolumn{3}{|c|}{ Another short-term hospital } \\
\hline All & $1.033(0.921-1.159)$ & 0.5702 \\
\hline \multicolumn{3}{|l|}{ Age (years) } \\
\hline $18-44$ & $0.901(0.729-1.113)$ & 0.3355 \\
\hline $45-64$ & $1.090(0.902-1.317)$ & 0.3696 \\
\hline
\end{tabular}

Table 3. Logistic Regression Analyses of the Temporal Trends of Hospital Disposition of ICU Admissions With Herpes Simplex Encephalitis for the Whole Cohort and Across Demographic Strata - (continued)

\begin{tabular}{|c|c|c|}
\hline Hospital disposition & Odds ratio $^{a}(95 \%$ CI $)$ & $\mathbf{P}$ \\
\hline$\geq 65$ & $1.119(0.906-1.382)$ & 0.2963 \\
\hline \multicolumn{3}{|l|}{ Gender } \\
\hline Female & $1.084(0.910-1.2910$ & 0.3646 \\
\hline Male & $0.9692(0.809-1.160)$ & \\
\hline \multicolumn{3}{|l|}{ Death $^{\mathrm{d}}$ or hospice } \\
\hline All & $1.014(0.956-1.075)$ & 0.6372 \\
\hline \multicolumn{3}{|l|}{ Age (years) } \\
\hline $18-44$ & $1.203(0.981-1.475)$ & 0.0752 \\
\hline $45-64$ & $0.990(0.887-1.105)$ & 0.8599 \\
\hline$\geq 65$ & $0.989(0.916-1.069)$ & 0.7956 \\
\hline \multicolumn{3}{|l|}{ Gender } \\
\hline Female & $1.017(0.935-1.108)$ & 0.6822 \\
\hline Male & $1.014(0.923-1.113)$ & 0.7707 \\
\hline \multicolumn{3}{|l|}{ Death $^{\mathrm{d}}$} \\
\hline All & $0.987(0.921-1.058)$ & 0.7149 \\
\hline \multicolumn{3}{|l|}{ Age (years) } \\
\hline $18-44$ & $1.127(0.917-1.385)$ & 0.2529 \\
\hline $45-64$ & $0.945(0.825-1.083)$ & 0.4171 \\
\hline$\geq 65$ & $0.970(0.887-1.061)$ & 0.5141 \\
\hline \multicolumn{3}{|l|}{ Gender } \\
\hline Female & $1.017(0.919-1.125)$ & 0.7419 \\
\hline Male & $0.974(0.874-1.085)$ & 0.6398 \\
\hline \multicolumn{3}{|l|}{ Hospice } \\
\hline All & $1.063(0.965-1.170)$ & 0.2110 \\
\hline \multicolumn{3}{|l|}{ Age (years) } \\
\hline $18-44$ & $0.983(0.951-1.201)$ & 0.9979 \\
\hline $45-64$ & $1.066(0.898-1.266)$ & 0.4596 \\
\hline$\geq 65$ & $1.028(0.913-1.157)$ & 0.6469 \\
\hline \multicolumn{3}{|l|}{ Gender } \\
\hline Female & $1.014(0.888-1.158)$ & 0.8354 \\
\hline Male & $1.097(0.935-1.286)$ & 0.2534 \\
\hline
\end{tabular}

aThe odds ratio results represent change per 1 year. As an illustrative example, the odds ratio of all home discharges among females $(0.925)$ means that the odds of all home discharges in this group decreased by $7.5 \%$ per year during the study period. ${ }^{b}$ Home health denotes those hospital discharges requiring home health services. ${ }^{\text {CPost-acute care }}$ denotes long-term care hospitals, skilled nursing facilities, and inpatient rehabilitation. ${ }^{d}$ Death denotes hospitalizations with in-hospital death. ICU: intensive care unit; $\mathrm{Cl}$ : confidence interval.

tween 2008 and 2016 from 39\% to 28.9\% (OR: 0.92 (95\% CI: $0.86-0.98) ; \mathrm{P}=0.0356)$, while the corresponding rate of routine home discharge decreased for the whole cohort and among females from $27.2 \%$ to $23.2 \%$ (OR: 0.93 (95\% CI: 0.89 - 0.98); $\mathrm{P}=0.0142)$, and from $31.7 \%$ to $21.1 \%(\mathrm{OR}: 0.90(95 \%$ 
CI: $0.83-0.98) ; P=0.0154)$, respectively. Finally, the rate of home discharge with home health rose among ICU hospitalizations aged 18 - 44 years from $0 \%$ to $22.7 \%$ (OR: 1.35 (95\% CI: 1.08 - 1.69); $\mathrm{P}=0.0083$ ).

\section{Discussion}

\section{Key findings}

In this large population-based study of adult HSVE hospitalizations most were admitted to ICU, with ICU utilization rising with age and increasing over time among older adults. Among ICU admissions, only about one-third of the patients were discharged home, while $40 \%$ were discharged to a post-acute care facility. Both transfers to post-acute care and short-term mortality rose progressively with age. When hospital disposition among ICU admissions was examined over time, rates of routine home discharge decreased for the whole cohort and female hospitalizations, while no significant change was observed in short-term mortality.

\section{Relationship to previous studies}

Only sparse data are available on the demand for critical care services among HSVE hospitalizations, while ICU utilization across specific patient subgroups has not been previously examined, to our knowledge. In two studies conducted in France, the ICU admission rates among HSVE hospitalizations were $71 \%$ during the years $1991-1998$ [13] and 32\% during 2010 - 2013 [8]. It is unclear whether the substantial decrease in ICU utilization over time in these studies reflects changes in practice-related factors or regional practice variations. There has been no report to show decreasing illness severity among adults with HSVE over time, which may have reduced the need for ICU admission, and the temporal trends of ICU utilization have not been previously reported.

The present study provides, to our knowledge, the first population-level examination of the patterns of demand for critical care services among adults hospitalized with HSVE. Most HSVE hospitalizations were critically ill, requiring admission to ICU, with a $60 \% / 40 \%$ distribution between ICU vs. non-ICU hospitalizations, respectively. The latter findings are similar to the reported findings from a 45-patient, single-center study in the USA (60\%) [14]. Our findings cannot be readily compared to those in France due to both possible differences in case mix and, importantly, due to the markedly lower availability of ICU beds in the latter [15].

The marked increase in ICU utilization with age likely reflects increased illness severity among older patients (see further discussion below). When examined over time, ICU utilization did not change, except for a rise among male HSVE hospitalizations and those aged 65 years or more. This increase in ICU admission rates may have been due to rising illness severity and complexity. However, there was no corresponding temporal change in hospital disposition in the latter two subgroups, though it is possible that the examined hospital dis- position domains were not sufficiently sensitive to the factors driving the observed ICU utilization changes.

Our findings demonstrate the large gaps in short-term outcomes between hospitalizations with HSVE who become critically and the reminder of affected patients, showing fourfold higher short-term mortality among the former. Similarly, outcomes were substantially worse among survivors, with the rates of discharge to a post-acute care facility $55 \%$ higher among ICU admissions. However, the patient-specific factors underlying progression to critical illness in some, but not other adults with HSVE remain unclear, precluding early targeted interventions. Only one study has compared, to our knowledge, the non-mortality outcomes at the time of hospital discharge between patients with HSVE who were admitted to ICU and those who were not. The investigators have shown in a single-center US cohort that unfavorable outcomes, based on the modified Rankin Scale were observed, were $77 \%$ more common among ICU admissions ( $77.8 \%$ vs. $44 \%$, respectively) [14].

Hospital mortality among ICU admissions in the present cohort is comparable to that described in a prior ICU-focused study $(12.4 \%$ vs. $11.9 \%)$ [8]. However, these figures underestimate the short-term mortality among critically ill patients with HSVE due to the considerable discharge to hospice in the USA, with the latter accounting for $34 \%$ of short-term mortality events in our cohort.

Rising age has been previously shown to be a major factor associated with adverse outcomes among patients with HSVE $[4,16]$. Our study provides the first population-level quantification of the profound worsening of short-term outcomes with age among the subset of critically ill patients with HSVE. Thus, short-term mortality occurred in over one out of four HSVE hospitalizations aged 65 or over admitted to ICU, and substantial residual morbidity, requiring transfer to a postacute care facility, was reported in one out of two, while only one in 10 had routine home discharge. The corresponding figures among those ICU admissions aged 18 - 44 years, were one in 10 , one in five, and one in two, respectively

Although the majority of HSVE hospitalizations admitted to ICU survived their hospital stay, over eight in 10 of all hospital deaths involved admission to ICU. This finding underscores the severity of HSVE among ICU admissions, as compared with critical illness in general, since ICU admission was reported in $59 \%$ of all hospital deaths in the general population in the USA [17].

Our study is the first, to our knowledge, to examine longitudinal, population-level, outcome trends of critically ill patients with HSVE. A key finding of the present study is that the short-term outcomes of critically ill patients with HSVE did not change over time or worsened in some subgroups. These findings contrast the progressive decrease in hospital mortality among critically ill patients in the general population [10]. The improved outcomes among the latter reflect the substantial improvement of critical care practice over the past decades and have occurred despite the concomitant rise in illness severity in the general population [10]. It is in this latter context that our findings of unchanged short-term mortality and high level of need for transfers to post-acute care of critically ill patients with HSVE are disconcerting. 
The factors underlying the latter findings are unclear and cannot be determined from claims-based data. However, it may be hypothesized that clinicians continue to face challenges in early consideration of possible HSVE upon hospital admission and with prompt initiation of acyclovir therapy. Delays in start of acyclovir therapy from the time of symptom onset [6] and since hospital admission [14] were found to be strongly associated with poor outcomes among patients with HSVE; and prior reports demonstrate lack material improvement in timeliness of acyclovir therapy. Thus, during 1991 - 1998 acyclovir therapy was initiated after 2 days or longer post hospital admission in 41\% [13] and was started on the first hospital day in 51.1\% during 1995 - 2013 (with acyclovir being started after 3 days in 25\%) [14]. A recent study on the factors associated with delays in starting acyclovir therapy found that severe underlying illness, alcohol abuse, and delay over 1 day since hospital admission to first brain imaging were all strongly and independently associated with delayed treatment [18]. In addition, finding of $<10$ leukocytes $/ \mathrm{mm}^{3}$ in the cerebrospinal fluid was associated with delay in start of acyclovir [18]. Clearly, clinician education to consider the possibility of HSVE, the atypical features of diagnostic findings, and the paramount importance of avoiding delay in starting empirical acyclovir therapy prior to results of CSF analysis are important steps in improving outcomes of affected patients. However, the rarity of HSVE and the multiple alternative explanations for patients' presentation make it challenging to change practice patterns. Finally, delayed admission to the ICU (that is, initial admission to a general ward, followed by latter transfer to ICU) was associated with poor functional outcomes among patients with HSVE, and those with delayed ICU admission had more commonly aspiration pneumonia and need for mechanical ventilation [9]. It is likely that the latter complications and, possibly, associated delays in start of acyclovir therapy have contributed to worse patient outcomes.

\section{Strengths and limitations}

The present study is the largest to date on adult critically patients with HSVE. The use of a statewide, all-payer, high-quality data set of consecutive hospitalizations allowed transcending of local variations in case mix and practice patterns. In addition, the large number of hospitalizations permitted more robust examination of the spatial and temporal aspects of the epidemiology and outcomes of ICU patients with HSVE.

However, our study has several important limitations, in addition to those mentioned earlier, related predominantly to its retrospective design and use of administrative data. First, because patients were identified based on ICD codes, there is a potential for misclassification, despite use of established search algorithms [19]. Second, as noted, the timeliness of acyclovir therapy and that of ICU admission, both of which could provide perspectives on the temporal changes in outcomes, could not be determined for the TIPUDF data set. Third, patients' functional state at the time of hospital discharge could not be determined and the proxy measures of hospital disposition provide limited information. Thus, additional studies using more granular data are needed. However, despite these limitations of the proxy measures, the findings on the spatial and temporal analyses of hospital outcomes provide a contemporary epidemiological perspective on the short-term outcomes of critically ill adults with HSVE. Last, the generalizability of our findings to other states, nationally, and to other countries is unknown.

\section{Conclusions}

The demand for critical care services is high among hospitalized adults with HSVE, increasing with age, and has been rising over time among older adults. Critically ill patients with HSVE have high short-term mortality and survivors have substantial residual morbidity, with older adults having especially poor short-term outcomes. The short-term outcomes among critically ill patients with HSVE did not improve over time and additional studies are urgently needed to identify scalable approaches to address modifiable outcome-associated factors in order to improve the prognosis of affected patients.

\section{Acknowledgments}

None to declare.

\section{Financial Disclosure}

None to declare.

\section{Conflict of Interest}

None to declare.

\section{Informed Consent}

Not applicable.

\section{References}

1. Bradshaw MJ, Venkatesan A. Herpes simplex virus-1 encephalitis in adults: pathophysiology, diagnosis, and management. Neurotherapeutics. 2016;13(3):493-508.

2. Boucher A, Herrmann JL, Morand P, Buzele R, Crabol Y, Stahl JP, Mailles A. Epidemiology of infectious encephalitis causes in 2016. Med Mal Infect. 2017;47(3):221235.

3. Whitley RJ, Soong SJ, Dolin R, Galasso GJ, Ch'ien LT, Alford CA. Adenine arabinoside therapy of biopsyproved herpes simplex encephalitis. National Institute of Allergy and Infectious Diseases collaborative antiviral study. N Engl J Med. 1977;297(6):289-294.

4. Modi S, Mahajan A, Dharaiya D, Varelas P, Mitsias P. Burden of herpes simplex virus encephalitis in the United States. J Neurol. 2017;264(6):1204-1208. 
5. Aurelius E, Johansson B, Skoldenberg B, Staland A, Forsgren M. Rapid diagnosis of herpes simplex encephalitis by nested polymerase chain reaction assay of cerebrospinal fluid. Lancet. 1991;337(8735):189-192.

6. Erdem H, Cag Y, Ozturk-Engin D, Defres S, Kaya S, Larsen L, Poljak M, et al. Results of a multinational study suggest the need for rapid diagnosis and early antiviral treatment at the onset of herpetic meningoencephalitis. Antimicrob Agents Chemother. 2015;59(6):3084-3089.

7. Mailles A, De Broucker T, Costanzo P, Martinez-Almoyna L, Vaillant V, Stahl JP, Steering C, et al. Long-term outcome of patients presenting with acute infectious encephalitis of various causes in France. Clin Infect Dis. 2012;54(10):1455-1464.

8. Jouan Y, Grammatico-Guillon L, Espitalier F, Cazals X, Francois P, Guillon A. Long-term outcome of severe herpes simplex encephalitis: a population-based observational study. Crit Care. 2015;19:345.

9. Jaquet P, de Montmollin E, Dupuis C, Sazio C, Conrad M, Susset V, Demeret S, et al. Functional outcomes in adult patients with herpes simplex encephalitis admitted to the ICU: a multicenter cohort study. Intensive Care Med. 2019;45(8):1103-1111.

10. Zimmerman JE, Kramer AA, Knaus WA. Changes in hospital mortality for United States intensive care unit admissions from 1988 to 2012. Crit Care. 2013;17(2):R81.

11. Texas inpatient public use data file. Texas Department of State Health Services, Center for Health Statistics, Austin, Texas. Available from: http://www.dshs.state.tx.us/ thcic/hospitals/Inpatientpudf.shtm.

12. Oud L, Watkins P. Contemporary trends of the epidemi- ology, clinical characteristics, and resource utilization of necrotizing fasciitis in Texas: a population-based cohort study. Crit Care Res Pract. 2015;2015:618067.

13. Raschilas F, Wolff M, Delatour F, Chaffaut C, De Broucker T, Chevret S, Lebon P, et al. Outcome of and prognostic factors for herpes simplex encephalitis in adult patients: results of a multicenter study. Clin Infect Dis. 2002;35(3):254-260.

14. Singh TD, Fugate JE, Hocker S, Wijdicks EFM, Aksamit AJ, Jr., Rabinstein AA. Predictors of outcome in HSV encephalitis. J Neurol. 2016;263(2):277-289.

15. Wunsch H, Angus DC, Harrison DA, Collange O, Fowler R, Hoste EA, de Keizer NF, et al. Variation in critical care services across North America and Western Europe. Crit Care Med. 2008;36(10):2787-2793, e2781-2789.

16. Jorgensen LK, Dalgaard LS, Ostergaard LJ, Norgaard M, Mogensen TH. Incidence and mortality of herpes simplex encephalitis in Denmark: A nationwide registry-based cohort study. J Infect. 2017;74(1):42-49.

17. Angus DC, Barnato AE, Linde-Zwirble WT, Weissfeld LA, Watson RS, Rickert T, Rubenfeld GD, et al. Use of intensive care at the end of life in the United States: an epidemiologic study. Crit Care Med. 2004;32(3):638-643.

18. Poissy J, Wolff M, Dewilde A, Rozenberg F, Raschilas F, Blas M, Georges H, et al. Factors associated with delay to acyclovir administration in 184 patients with herpes simplex virus encephalitis. Clin Microbiol Infect. 2009;15(6):560-564.

19. George BP, Schneider EB, Venkatesan A. Encephalitis hospitalization rates and inpatient mortality in the United States, 2000-2010. PLoS One. 2014;9(9):e104169. 